\title{
Gap Minimization for Peer-Evaluation in DEA Cross-Efficiency
}

\author{
Anrong Yang, ${ }^{1}$ Zigang Zhang, ${ }^{1}$ Yishi Zhang, ${ }^{1,2}$ and Dunliang Chen ${ }^{1}$ \\ ${ }^{1}$ School of Management, Huazhong University of Science and Technology, 1037 Luoyu Road, Hongshan District, Wuhan 430074, China \\ ${ }^{2}$ Wisconsin School of Business, University of Wisconsin-Madison, Madison, WI 53706, USA \\ Correspondence should be addressed to Yishi Zhang; z14@hust.edu.cn
}

Received 28 February 2014; Revised 19 July 2014; Accepted 20 July 2014; Published 6 August 2014

Academic Editor: Farhad Hosseinzadeh Lotfi

Copyright (C) 2014 Anrong Yang et al. This is an open access article distributed under the Creative Commons Attribution License, which permits unrestricted use, distribution, and reproduction in any medium, provided the original work is properly cited.

\begin{abstract}
Cross-efficiency evaluation is an effective and widely used method for ranking decision making units (DMUs) in data envelopment analysis (DEA). Gap minimization criterion is introduced in aggressive and benevolent cross-efficiency methods to avoid possible extreme efficiency from peer-evaluation and to get equitable results. On the basis of this criterion, a weighted cross-efficiency method with similarity distance that, respectively, considers the aggressive and the benevolent formulations is proposed to determine cross-efficiency. The weights of the cross-evaluation determined by this method are positively influenced by selfevaluation and thus are propitious to resolving conflict. Numerical demonstration reveals the feasibility of the proposed method.
\end{abstract}

\section{Introduction}

Data envelopment analysis (DEA) is an effective and widely used nonparametric method for assessing the relative efficiency of a set of decision making units (DMUs) with structures of multiple inputs and outputs. Using the traditional DEA models like CCR model proposed by Charnes et al. [1] and BCC model by Banker et al. [2], DMUs can be divided into efficient and inefficient groups. DEA is a typical application of linear programming and has already been widely used for solving many real world problems [3-6]. While in practice, there is often a need to discriminate efficient DMUs. Besides, DEA may result in unrealistic weight distribution, thus leading to the efficiency results being not equitable and acceptable in some cases. A series of approaches have been proposed to rank DMUs and to overcome the shortcomings of traditional DEA methods [7-17].

Cross-efficiency method originated by Sexton et al. [18] extends the basic DEA methods and thus can rank DMUs according to their efficiency scores. In addition, it can avoid unrealistic DEA weighting schemes by incorporating additional weight restrictions into DEA. The core idea of the cross-efficiency is to evaluate each DMU according to both its and peers' optimal input and output weights. The efficiency scores of a DMU will thus be calculated $n$ times and the average score of self- and peer-evaluations will be chosen as its ranking score. However, this may fall into a dilemma of multiple solutions [19]. Doyle and Green [20] propose an aggressive cross-efficiency method and a benevolent one to avoid the problem. They introduce secondary objective functions to select the optimal weights minimizing and maximizing the sum of the outputs of other DMUs, respectively. Wang and Chin [16] propose a neutral crossefficiency method to reduce the number of zero weights of the outputs. The cross-efficiency scores are determined by the profiles of weights that are used. To effectively select weights from multiple optimal solutions, goal programming formulations are considered to be an effective method in recent literature (e.g., SWAT proposed by Jahanshahloo et al. [21] and the novel secondary objective function proposed by Hosseinzadeh-Lotfi et al. [22]). Many alternative secondary goals for the purpose of cross-efficiency are proposed in the literature, where minimizing the summation of deviations from the ideal point and minimizing the maximum quantity among all deviation variables are typically applied in [23, 24]. In addition, multicriteria method proposed by $\mathrm{Li}$ and Reeves [23] and its extensions like preemptive programming formulation by Bal and Örkcü [8] and weighted programming formulation by [9] have the ability to select weights with realistic weight distribution. Örkcü and Bal [14] refine 
goal programming models in the cross-efficiency evaluation. Recently, Wang et al. [17] propose a simultaneously input- and output-oriented weight determination cross-efficiency model for reducing the number of zero weights of the inputs.

In this paper, we mainly focus on the promotion of the acceptance of the cross-efficiency ranking results for each DMU. We introduce a regularization term to minimize the gap between the upper and lower bounds of the peer-evaluation scores. In addition, we evaluate DMUs in a more neutral manner both considering the pessimistic and optimistic attitudes from the aggressive and benevolent methods and propose a weighted neutral cross-efficiency method to determine the cross-efficiency scores according to the similarity distances between DMUs. Our method has a greater ability to discriminate DEA-efficient and DEAinefficient DMUs compared to the extant cross-efficiency methods. In addition, the gap minimization criterion and the manner of determining the weights for each cross-efficiency score in our method are propitious to resolving conflict among DMUs during cross-evaluation process.

The rest of this paper is organized as follows. Section 2 briefly introduces the aggressive and benevolent crossefficiency models. Section 3 proposes a secondary goal to minimize the gap between the upper and lower bounds of the peer-evaluation scores and a new weighted method for neutral DEA cross-efficiency evaluation based on the similarity distance between pairs of DMUs. Then Section 4 demonstrates the feasibility of proposed method with a numerical example. Section 5 finally summarizes the concluding remarks.

\section{Aggressive and Benevolent Cross-Efficiency Models}

Let there be $n$ DMUs where $\mathrm{DMU}_{j}(j=1,2, \ldots, n)$ uses $m$ inputs $x_{i j}(i=1,2, \ldots, m)$ to produce $s$ outputs $y_{r j}(r=$ $1,2, \ldots, s)$. And suppose that all input and output indices are nonnegative constant numbers. Then for a given $\mathrm{DMU}_{p}(1 \leq$ $p \leq n)$, the relative efficiency score under the CCR model is determined by solving the following linear programming [1]:

$$
\begin{array}{ll}
\max & \theta_{p p}:=\sum_{r=1}^{s} u_{r p} y_{r p} \\
\text { s.t. } & \sum_{i=1}^{m} v_{i p} x_{i p}=1 \\
& \sum_{i=1}^{m} v_{i p} x_{i j}-\sum_{r=1}^{s} u_{r p} y_{r j} \geq 0, \quad j=1,2, \ldots, n \\
& u_{r p} \geq 0, \quad v_{i p} \geq 0, \quad r=1,2, \ldots, s ; \quad i=1,2, \ldots, m,
\end{array}
$$

where $v_{i p}(i=1,2, \ldots, m)$ and $u_{r p}(r=1,2, \ldots, s)$ are the weights assigned to $i$ th input and $r$ th output, respectively. Let $v_{i p}^{*}$ and $u_{r p}^{*}$ be the optimal solutions to model (1); then $\theta_{p p}^{*}=$ $\sum_{r=1}^{s} u_{r p}^{*} y_{r p}$ is the CCR-efficiency of $\mathrm{DMU}_{p}$ and reflects the self-evaluation of this unit. $\mathrm{DMU}_{p}$ is considered to be efficient if $\theta_{p p}^{*}=1$; otherwise, it is referred to as nonefficient.

As an extension to basic DEA models, cross-efficiency evaluation not only generates a ranking among the DMUs according to their efficiency but also removes unrealistic DEA weighting schemes without requiring any additional information on weight restrictions. The cross-efficiency $\bar{\theta}_{p}^{*}$ of $\mathrm{DMU}_{p}$ which contains $\mathrm{DMU}_{j}$ 's peer-evaluation to $\mathrm{DMU}_{p}(j=1, \ldots, n ; j \neq p)$ is obtained as

$$
\begin{gathered}
\theta_{j p}^{*}=\frac{\sum_{r=1}^{s} u_{r j}^{*} y_{r p}}{\sum_{i=1}^{m} v_{i j}^{*} x_{i p}}, \quad j=1, \ldots, n ; j \neq p, \\
\bar{\theta}_{p}^{*}=\frac{1}{n}\left(\sum_{j=1, j \neq p}^{n} \theta_{j p}^{*}+\theta_{p p}^{*}\right) .
\end{gathered}
$$

Using the average cross-efficiency values, all of the DMUs can be compared and ranked. However, a factor that possibly reduces the usefulness of the cross-efficiency evaluation method is that the cross-efficiency results may not be unique due to the presence of alternate optima. To resolve the problem of having multiple optimal solutions from model (1) so as to keep $\theta_{j p}^{*}$ unchanged, Sexton et al. [18] introduce a secondary goal to select the optimal input and output weights from multiple optimal solutions while keeping the CCR efficiency $\theta_{p p}^{*}$ unchanged. The most representative secondary goals are shown in the following models $[18,20]$ :

$$
\begin{array}{ll}
\min \text { or } \max & \sum_{r=1}^{s}\left(u_{r p} \sum_{j=1, j \neq p}^{n} y_{r j}\right) \\
\text { s.t. } & \sum_{i=1}^{m} v_{i p}\left(\sum_{j=1, j \neq p}^{n} x_{i j}\right)=1 \\
& \sum_{r=1}^{s} u_{r p} y_{r p}-\theta_{p p}^{*} \sum_{i=1}^{m} v_{i p} x_{i p}=0 \\
& \sum_{i=1}^{m} v_{i p} x_{i j}-\sum_{r=1}^{s} u_{r p} y_{r j} \geq 0, \\
& j=1,2, \ldots, n ; \quad j \neq p \\
& u_{r p}, v_{i p} \geq 0, \quad r=1,2, \ldots, s ; \quad i=1,2, \ldots, m,
\end{array}
$$

where $\theta_{p p}^{*}$ is the efficiency value for $\mathrm{DMU}_{p}$ obtained from model (1). Model (4) with a min-objective function is known as the aggressive efficiency model, which minimizes the cross-efficiencies of other DMUs while preserving the efficiency of itself under evaluation. Model (4) with a maxobjective function is known as the benevolent efficiency model, which maximizes the cross-efficiencies of other DMUs while preserving the efficiency of itself under evaluation. 


\section{A Weighted Neutral Cross-Efficiency Method}

3.1. Gap Minimization Criterion for Peer-Evaluation. In the case of the benevolent model, the idea is to identify the optimal weights that not only maximize the efficiency of a particular DMU under evaluation but, at the same time, maximize the average efficiency of other DMUs. In the case of the aggressive model, one seeks weights that minimize the average efficiency of those other units. Although these two goals have been proposed as a remedy for the issue of nonuniqueness, they only focus on maximizing or minimizing the value of the efficiencies of self- and peer-evaluations but never consider the rationality of possible extreme results of peer-evaluation, for example, selecting weights making the value of peer-evaluation for a certain DMU too small or too large. This may result in dispute and impair the rationality of the selected weights and the corresponding peer-efficiency scores, especially for the DMUs in a cooperative mode. To this end, the objective function of the cross-efficiency model should not only consider the issue of nonuniqueness but also take into account the rationality of the results of peerevaluation. For the aggressive and the benevolent models, the extreme results of peer-evaluation can be partially avoided by introducing an additional goal or a regularization term focusing on such issue. In this paper, we try to avoid the extreme positions by means of minimizing the gap between the extreme large value and the extreme small one of the peerevaluation. This can be formed as

$$
\min \left(\max _{j=1, \ldots, n, j \neq p} \sum_{r=1}^{s} u_{r p} y_{r j}-\min _{k=1, \ldots, n, k \neq p} \sum_{r=1}^{s} u_{r p} y_{r k}\right)
$$

Hence, we apply (5) as the regularization term and add it as a part of the objective function for both the aggressive and the benevolent models. We show the modified aggressive and benevolent models, respectively, as

$$
\begin{array}{ll}
\min & \sum_{r=1}^{s}\left(u_{r p} \sum_{j=1, j \neq p}^{n} y_{r j}\right) \\
& +\rho \cdot\left(\max _{j=1, \ldots, n, j \neq p} \sum_{r=1}^{s} u_{r p} y_{r j}-\min _{k=1, \ldots, n, k \neq p} \sum_{r=1}^{s} u_{r p} y_{r k}\right) \\
\text { s.t. } \quad & \sum_{i=1}^{m} v_{i p}\left(\sum_{j=1, j \neq p}^{n} x_{i j}\right)=1 \\
& \sum_{r=1}^{s} u_{r p} y_{r p}-\theta_{p p}^{*} \sum_{i=1}^{m} v_{i p} x_{i p}=0 \\
& \sum_{i=1}^{m} v_{i p} x_{i j}-\sum_{r=1}^{s} u_{r p} y_{r j} \geq 0, \\
& j=1,2, \ldots, n ; \quad j \neq p \\
& u_{r p}, v_{i p} \geq 0, \quad r=1,2, \ldots, s ; \quad i=1,2, \ldots, m,
\end{array}
$$

$$
\begin{array}{ll}
\max & \sum_{r=1}^{s}\left(u_{r p} \sum_{j=1, j \neq p}^{n} y_{r j}\right) \\
& -\rho \cdot\left(\max _{j=1, \ldots, n, j \neq p} \sum_{r=1}^{s} u_{r p} y_{r j}-\min _{k=1, \ldots, n, k \neq p} \sum_{r=1}^{s} u_{r p} y_{r k}\right) \\
\text { s.t. } \quad & \sum_{i=1}^{m} v_{i p}\left(\sum_{j=1, j \neq p}^{n} x_{i j}\right)=1 \\
& \sum_{r=1}^{s} u_{r p} y_{r p}-\theta_{p p}^{*} \sum_{i=1}^{m} v_{i p} x_{i p}=0 \\
& \sum_{i=1}^{m} v_{i p} x_{i j}-\sum_{r=1}^{s} u_{r p} y_{r j} \geq 0, \quad j=1,2, \ldots, n ; j \neq p \\
& u_{r p}, v_{i p} \geq 0, \quad r=1,2, \ldots, s ; \quad i=1,2, \ldots, m,
\end{array}
$$

where $\rho \in[0,+\infty)$ is the importance weight of the regularization term (5) in the objective functions of models (6) and (7). Note that $\rho=0$ makes models (6) and (7) the same as the original aggressive model and the original benevolent one, respectively. If $\rho$ is large enough, (6) and (7) are equivalent to the model that always focuses on the gap between extremes.

Models (6) and (7) can be expressed equivalently in the following forms, respectively:

$$
\begin{array}{ll}
\min & \sum_{r=1}^{s}\left(u_{r p} \sum_{j=1, j \neq p}^{n} y_{r j}\right)+\rho \cdot(\bar{\lambda}-\underline{\lambda}) \\
\text { s.t. } \quad & \sum_{i=1}^{m} v_{i p}\left(\sum_{j=1, j \neq p}^{n} x_{i j}\right)=1 \\
& \sum_{r=1}^{s} u_{r p} y_{r p}-\theta_{p p}^{*} \sum_{i=1}^{m} v_{i p} x_{i p}=0 \\
& \sum_{i=1}^{m} v_{i p} x_{i j}-\sum_{r=1}^{s} u_{r p} y_{r j} \geq 0, \quad j=1,2, \ldots, n ; j \neq p \\
& \bar{\lambda}-\sum_{r=1}^{s} u_{r p} y_{r j} \geq 0, \quad j=1,2, \ldots, n ; j \neq p \\
& \sum_{r=1}^{s} u_{r p} y_{r j}-\underline{\lambda} \geq 0, \quad j=1,2, \ldots, n ; j \neq p \\
& u_{r p}, v_{i p} \geq 0, \quad r=1,2, \ldots, s ; \quad i=1,2, \ldots, m,
\end{array}
$$




$$
\begin{array}{ll}
\max & \sum_{r=1}^{s}\left(u_{r p} \sum_{j=1, j \neq p}^{n} y_{r j}\right)-\rho \cdot(\bar{\lambda}-\underline{\lambda}) \\
\text { s.t. } \quad & \sum_{i=1}^{m} v_{i p}\left(\sum_{j=1, j \neq p}^{n} x_{i j}\right)=1 \\
& \sum_{r=1}^{s} u_{r p} y_{r p}-\theta_{p p}^{*} \sum_{i=1}^{m} v_{i p} x_{i p}=0 \\
& \sum_{i=1}^{m} v_{i p} x_{i j}-\sum_{r=1}^{s} u_{r p} y_{r j} \geq 0, \quad j=1,2, \ldots, n ; j \neq p \\
& \bar{\lambda}-\sum_{r=1}^{s} u_{r p} y_{r j} \geq 0, \quad j=1,2, \ldots, n ; j \neq p \\
& \sum_{r=1}^{s} u_{r p} y_{r j}-\underline{\lambda} \geq 0, \quad j=1,2, \ldots, n ; j \neq p \\
& u_{r p}, v_{i p} \geq 0, \quad r=1,2, \ldots, s ; \quad i=1,2, \ldots, m,
\end{array}
$$

where $\bar{\lambda}$ and $\underline{\lambda}$ are the upper and lower bounds of $\sum_{r=1}^{s} u_{r p} y_{r j}$, respectively.

3.2. A Neutral Method for Peer-Efficiency Determination. In order to eliminate bias to other DMUs brought by both of the aggressive goal and the benevolent one as well as keep the objectivity of the evaluation process, we try to evaluate DMUs in a more neutral manner and to reduce conflict and disputes by applying both of the models. That is, we can make compromised results between the aggressive and the benevolent formulations if the final peer-efficiency scores are determined in terms of such two formulations. To illustrate this, for $\mathrm{DMU}_{p}$, we denote its self- and peer-efficiency scores $\gamma_{p p}^{*}$ and $\gamma_{j p}^{*}(j=1, \ldots, n, j \neq p)$, respectively, by solving models (8) and (3) and self- and peer-efficiency scores $\delta_{p p}^{*}$ and $\delta_{j p}^{*}(j=1, \ldots, n, j \neq p)$, respectively, by solving models (9) and (3). We hence have the following convex combinatorial expression for peer-efficiency score $\theta_{j p}^{*}$ :

$$
\theta_{j p}^{*}=\alpha \cdot \gamma_{j p}^{*}+\beta \cdot \delta_{j p}^{*}, \quad 1 \leq j \leq n,
$$

where the convex combinatorial coefficients $\alpha$ and $\beta$ satisfy $\alpha+\beta=1$ and $\alpha \geq 0, \beta \geq 0$. Such a combination effectively balances the two extreme attitudes from the aggressive and the benevolent formulations and thus results in a more "neutral" peer-efficiency score. Thus proposed method can be taken as a neutral cross-efficiency method. For $\theta_{j p}^{*}$, we have the following proposition.

Proposition 1. $\theta_{j p}^{*} \leq \theta_{p p}^{*}(j=1, \ldots, n)$.

Proof. Let the optimum solutions of $\mathrm{DMU}_{j}$ from model (8) be $v_{i j}^{*}(i=1,2, \ldots, m)$ and $u_{r j}^{*}(r=1,2, \ldots, s)$, respectively, and let those from model $(9)$ be $v_{i j}^{*}(i=1,2, \ldots, m)$ and $\mu_{r j}^{*}(r=1,2, \ldots, s)$, respectively. Note that both the optimum solutions from models (8) and (9) satisfy the following constraint:

$$
\sum_{r=1}^{s} u_{r p} y_{r p}-\theta_{p p}^{*} \sum_{i=1}^{m} v_{i p} x_{i p}=0 .
$$

Recall that $\theta_{p p}^{*}$ denotes the optimum value of the CCR model (i.e., model (1)). This constraint can be rewrite as

$$
\frac{\sum_{r=1}^{s} u_{r p} y_{r p}}{\sum_{i=1}^{m} v_{i p} x_{i p}}=\theta_{p p}^{*} .
$$

Hence, feasible solutions from models (8) and (9) are always optimum to the CCR model. Thus we have

$$
\begin{aligned}
& \gamma_{j p}^{*}=\frac{\sum_{r=1}^{s} u_{r j}^{*} y_{r p}}{\sum_{i=1}^{m} v_{i j}^{*} x_{i p}} \leq \frac{\sum_{r=1}^{s} u_{r p}^{*} y_{r p}}{\sum_{i=1}^{m} v_{i p}^{*} x_{i p}}=\theta_{p p}^{*}, \quad j=1, \ldots, n, \\
& \delta_{j p}^{*}=\frac{\sum_{r=1}^{s} \mu_{r j}^{*} y_{r p}}{\sum_{i=1}^{m} v_{i j}^{*} x_{i p}} \leq \frac{\sum_{r=1}^{s} \mu_{r p}^{*} y_{r p}}{\sum_{i=1}^{m} v_{i p}^{*} x_{i p}}=\theta_{p p}^{*}, \quad j=1, \ldots, n .
\end{aligned}
$$

Meanwhile, $\theta_{j p}^{*}=\alpha \cdot \gamma_{j p}^{*}+\beta \cdot \delta_{j p}^{*}(\alpha+\beta=1)$ implies

$$
\min \left(\gamma_{j p}^{*}, \delta_{j p}^{*}\right) \leq \theta_{j p}^{*} \leq \max \left(\gamma_{j p}^{*}, \delta_{j p}^{*}\right)
$$

With (13) and (14), we have $\theta_{j p}^{*} \leq \theta_{p p}^{*}(j=1, \ldots, n)$.

To reduce inconsistency between peer-evaluation and self-evaluation, we determine $\alpha$ and $\beta$ from the viewpoint of efficiency distance between pairs of DMUs. From an individual's viewpoint, DMUs with more similar efficiency scores are more important to be referred during the crossefficiency evaluation process. More specifically, for $\mathrm{DMU}_{j}$ and $\mathrm{DMU}_{p}$, the more $\gamma_{j p}^{*}\left(\delta_{j p}^{*}\right)$ is similar to $\theta_{p p}^{*}$, the more likely the profile of weights of inputs and outputs of $\mathrm{DMU}_{j}$ is to be accepted by $\mathrm{DMU}_{p}$. Thus, we define the distance from $\gamma_{j p}^{*}$ to $\theta_{p p}^{*}$ as

$$
d_{\gamma}(j, p)=\left|\gamma_{j p}^{*}-\theta_{p p}^{*}\right|
$$

and that from $\delta_{j p}^{*}$ to $\theta_{p p}^{*}$ as

$$
d_{\delta}(j, p)=\left|\delta_{j p}^{*}-\theta_{p p}^{*}\right| .
$$

Note that $d_{\gamma}(j, p) \neq d_{\gamma}(p, j)$ and $d_{\delta}(j, p) \neq d_{\delta}(p, j)$ in most cases. Since small (large) value of $d_{\gamma}(j, p)$ implies strong (weak) similarity between $\mathrm{DMU}_{j}$ and $\mathrm{DMU}_{p}$, we hence introduce a total distance $d_{\text {total }}(j, p)$ as

$$
d_{\text {total }}(j, p)=d_{\gamma}(j, p)+d_{\delta}(j, p),
$$

and conflict reduction may be implemented by keeping $\alpha$ and $\beta$ in proportion to $\left[d_{\text {total }}(j, p)-d_{\gamma}(j, p)\right]$ and $\left[d_{\text {total }}(j, p)-\right.$ $\left.d_{\delta}(j, p)\right]$, respectively; that is,

$$
\begin{aligned}
& \alpha \propto d_{\delta}(j, p), \\
& \beta \propto d_{\gamma}(j, p) .
\end{aligned}
$$


To determine the value of $\alpha$ and $\beta$, normalization is needed to make them satisfy $\alpha+\beta=1$. Hence we have

$$
\begin{aligned}
& \alpha=\frac{d_{\delta}(j, p)}{d_{\text {total }}(j, p)}=\frac{\left|\delta_{j p}^{*}-\theta_{p p}^{*}\right|}{\left|\gamma_{j p}^{*}-\theta_{p p}^{*}\right|+\left|\delta_{j p}^{*}-\theta_{p p}^{*}\right|}, \\
& \beta=\frac{d_{\gamma}(j, p)}{d_{\text {total }}(j, p)}=\frac{\left|\gamma_{j p}^{*}-\theta_{p p}^{*}\right|}{\left|\gamma_{j p}^{*}-\theta_{p p}^{*}\right|+\left|\delta_{j p}^{*}-\theta_{p p}^{*}\right|} .
\end{aligned}
$$

With Proposition 1, it is easy to find that (19) can be transferred into easy-to-solve forms as follows:

$$
\begin{aligned}
& \alpha=\frac{\theta_{p p}^{*}-\delta_{j p}^{*}}{2 \theta_{p p}^{*}-\delta_{j p}^{*}-\gamma_{j p}^{*}}, \\
& \beta=\frac{\theta_{p p}^{*}-\gamma_{j p}^{*}}{2 \theta_{p p}^{*}-\delta_{j p}^{*}-\gamma_{j p}^{*}} .
\end{aligned}
$$

3.3. Cross-Evaluation Based on Similarity Distance. To further reduce the inconsistency between peer-evaluation and self-evaluation, we also determine the weight for crossevaluation $\omega_{j p}$ from the viewpoint of efficiency distance between pairs of DMUs. We define the cross-evaluationbased distance from $\theta_{j p}^{*}$ to $\theta_{p p}^{*}$ as

$$
d(j, p)=\left|\theta_{j p}^{*}-\theta_{p p}^{*}\right|, \quad 1 \leq j \leq n .
$$

Let $d_{\max }=\max _{j} d(j, p)$; the cross-efficiency weight of $\theta_{j p}^{*}$ should be proportional to $\left(d_{\max }-d(j, p)\right)$. Taking normalization into consideration, we apply

$$
\omega_{j p}=\frac{d_{\max }-d(j, p)}{\sum_{i=1}^{n}\left(d_{\max }-d(i, p)\right)}
$$

as the cross-efficiency weight of $\theta_{j p}^{*}$. To make (22) easy to solve, we have (with Proposition 1)

$$
d_{\max }=\max _{j}\left|\theta_{j p}^{*}-\theta_{p p}^{*}\right|=\theta_{p p}^{*}-\min _{j} \theta_{j p}^{*} .
$$

Let $\theta_{\min }^{*}=\min _{j} \theta_{j p}^{*},(22)$ be equivalent to

$$
\omega_{j p}=\frac{\theta_{j p}^{*}-\theta_{\min }^{*}}{\sum_{i=1}^{n} \theta_{i p}^{*}-n \theta_{\min }^{*}} .
$$

And finally we have

$$
\begin{gathered}
\omega_{j p}=\frac{\theta_{j p}^{*}-\theta_{\min }^{*}}{\sum_{i=1}^{n} \theta_{i p}^{*}-n \theta_{\min }^{*}}, \quad j=1, \ldots, n, \\
\bar{\theta}_{p}^{*}=\sum_{j=1}^{n} \omega_{j p} \theta_{j p}^{*} .
\end{gathered}
$$

We use (10) in neither aggressive nor benevolent manners but a more neutral way to calculate peer-evaluation and use (26) to get the weighted cross-efficiency according to the distance
TABLE 1: Description of the inputs and the outputs of 14 passenger airlines.

\begin{tabular}{lcl}
\hline & Indices & Description \\
\hline \multirow{3}{*}{ Inputs } & $x_{1}$ & Aircraft capacity in ton kilometers \\
& $x_{2}$ & Operating cost \\
& $x_{3}$ & Nonflight assets \\
\hline \multirow{2}{*}{ Outputs } & $y_{1}$ & Passenger kilometers \\
& $y_{2}$ & Nonpassenger revenue \\
\hline
\end{tabular}

between the currently evaluated DMU and peers. Peers' evaluation with different weights according to the distance can partially mitigate conflict among DMUs because the evaluation perspective of peers with short distance is more likely to be accepted by the currently evaluated DMU. We use a numeric example in the following section to illustrate the advantages of our method.

\section{An Illustrative Example}

In this section, our method is illustrated with a numerical example. In the example, fourteen major international passenger airlines (DMUs) are needed to be evaluated in terms of three inputs $\left(x_{1}, x_{2}, x_{3}\right)$ and two outputs $\left(y_{1}, y_{2}\right)$ [25]. Description of the inputs and the outputs is shown in Table 1. We apply in this section the aggressive and benevolent models (shown in model (4)) as the comparison models. We also apply CCR model as the basic DEA model and report their CCR-efficiency scores for some necessary analysis. Recall that the importance weight $\rho$ in models (8) and (9) reflects the magnitude of the gap minimization goal (see (5)) and thus may result in various solutions if its value changes. To balance the importance of the gap minimization goal, $\rho$ should be neither too large nor too small. In this example, we heuristically set $\rho=0.2$.

Table 2 shows the input and output data, proposed neutral weighted cross-efficiency results, the aggressive and benevolent cross-efficiency results (shown in " $\theta$ " " and "\#" columns), and the CCR-efficiency results (shown in " $\theta_{\mathrm{CCR}}^{*}$ " column) of the fourteen passenger airlines. Efficiency scores from proposed method, aggressive and benevolent methods, and CCR are also shown in Figure 1. It can be seen that seven DMUs are CCR-efficient (i.e., the 5th, 7th, and 1014th DMUs). For these CCR-efficient DMUs, three of the cross-efficiency methods all fully rank them with peer- and self-evaluation scores. Compared with the aggressive and benevolent methods, proposed method produces different efficiency rankings for 14 international passenger airlines. Specifically speaking, all CCR-efficient DMUs are top-ranked from top 1 to top 7, respectively, using proposed method, whereas neither aggressive nor benevolent methods can achieve this. For example, at least one CCR-inefficient DMU (e.g., $\mathrm{DMU}_{4}$ evaluated by both aggressive and benevolent methods) is ranked as one of the top 7 DMUs while at least one CCR-efficient DMU (e.g., DMU 7 evaluated by aggressive method and $\mathrm{DMU}_{10}$ evaluated by benevolent method) is out of them. For CCR-efficient DMUs, proposed method is 
TABLE 2: Data, efficiency scores, and ranks for 14 passenger airlines using different cross-efficiency models.

\begin{tabular}{|c|c|c|c|c|c|c|c|c|c|c|c|c|}
\hline \multirow{2}{*}{ DMU } & \multirow{2}{*}{$x_{1}$} & \multirow[b]{2}{*}{$x_{2}$} & \multirow{2}{*}{$x_{3}$} & \multirow{2}{*}{$y_{1}$} & \multirow{2}{*}{$y_{2}$} & \multirow{2}{*}{$\theta_{\mathrm{CCR}}^{*}$} & \multicolumn{2}{|c|}{ Aggressive } & \multicolumn{2}{|c|}{ Benevolent } & \multicolumn{2}{|c|}{ Proposed } \\
\hline & & & & & & & $\bar{\theta}^{*}$ & $\#$ & $\bar{\theta}^{*}$ & $\#$ & & \# \\
\hline 1 & 5723 & 3239 & 2003 & 26667 & 697 & 0.8681 & 0.5990 & 12 & 0.7541 & 12 & 0.7175 & 13 \\
\hline 2 & 5895 & 4225 & 4557 & 3081 & 539 & 0.3379 & 0.1652 & 14 & 0.1894 & 14 & 0.2328 & 14 \\
\hline 3 & 24099 & 9560 & 6267 & 124055 & 1266 & 0.9475 & 0.6226 & 11 & 0.7678 & 9 & 0.7549 & 11 \\
\hline 4 & 13565 & 7499 & 3213 & 64734 & 1563 & 0.9581 & 0.6734 & 7 & 0.8222 & 6 & 0.7848 & 9 \\
\hline 5 & 5183 & 1880 & 783 & 23604 & 513 & 1 & 0.7983 & 1 & 0.8912 & 3 & 0.8831 & 4 \\
\hline 6 & 19080 & 8032 & 3272 & 95011 & 572 & 0.9766 & 0.6385 & 9 & 0.7554 & 11 & 0.7909 & 8 \\
\hline 7 & 4603 & 3457 & 2360 & 22112 & 969 & 1 & 0.6478 & 8 & 0.8214 & 7 & 0.8442 & 6 \\
\hline 8 & 12097 & 6779 & 6474 & 52363 & 2001 & 0.8588 & 0.5855 & 13 & 0.7242 & 13 & 0.7356 & 12 \\
\hline 9 & 6587 & 3341 & 3581 & 26504 & 1297 & 0.9477 & 0.6309 & 10 & 0.7590 & 10 & 0.7818 & 10 \\
\hline 10 & 5654 & 1878 & 1916 & 19277 & 972 & 1 & 0.6813 & 6 & 0.7803 & 8 & 0.8176 & 7 \\
\hline 11 & 12559 & 8098 & 3310 & 41925 & 3398 & 1 & 0.7742 & 2 & 0.9193 & 1 & 0.9391 & 1 \\
\hline 12 & 5728 & 2481 & 2254 & 27754 & 982 & 1 & 0.7314 & 5 & 0.8850 & 4 & 0.8906 & 3 \\
\hline 13 & 4715 & 1792 & 2485 & 31332 & 543 & 1 & 0.7503 & 3 & 0.9190 & 2 & 0.9076 & 2 \\
\hline 14 & 22793 & 9874 & 4145 & 122528 & 1404 & 1 & 0.7316 & 4 & 0.8659 & 5 & 0.8795 & 5 \\
\hline
\end{tabular}

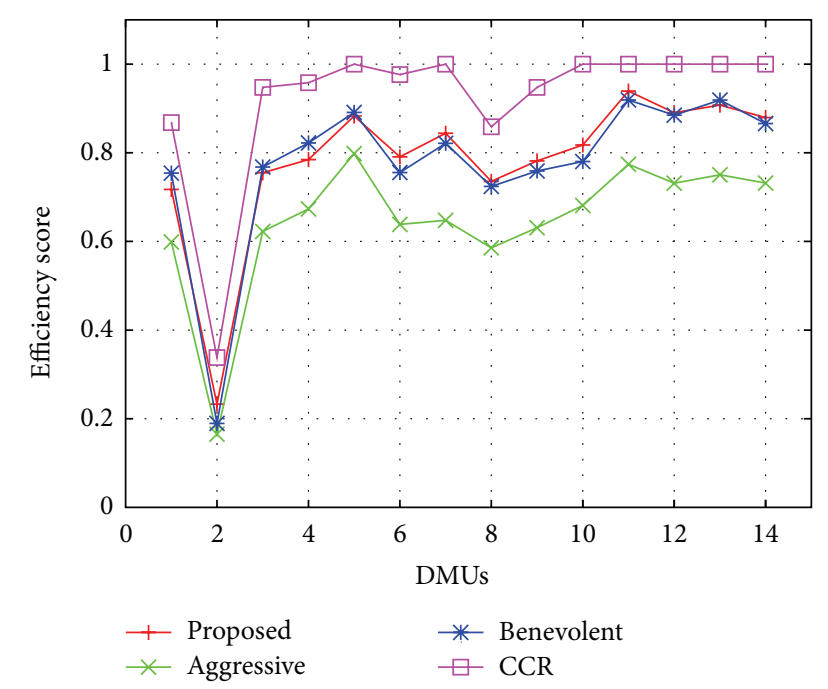

FIGURE 1: Efficiency scores from proposed, aggressive, benevolent model and CCR methods.

flexible in balancing the different attitudes from aggressive and benevolent methods, for example, the results of $\mathrm{DMU}_{10}$. For CCR-inefficient DMUs, it is less likely that proposed method regards them to be more efficient than CCR-efficient ones. This illustrates that proposed method is effective to discriminate CCR-efficient from CCR-inefficient DMUs and to evaluate and rank DMUs from a more neutral perspective. We believe that our method is more equitable and reliable than the methods that rank DMUs according to either the aggressive or the benevolent cross-efficiency scores, for the gap minimization goal to avoid extreme efficiency scores is more acceptable and an integration of both pessimistic and optimistic attitudes is more effective to mitigate conflict especially for DMUs in a cooperative mode.

\section{Conclusions}

Cross-efficiency evaluation is an important method for comparing and ranking DMUs. Extant research has made efforts to tackle the shortcomings of cross-efficiency but seldom considers how to effectively discriminate DEAefficient from DEA-inefficient DMUs and to reduce conflict during the cross-evaluation process. Based on two typical cross-efficiency methods, we propose a secondary goal to minimize the gap between the upper and lower bounds of the peer-evaluation scores and a weighted neutral crossefficiency method to determine cross-efficiency of each DMU with a combination of its aggressive and benevolent crossefficiency. A numerical example is conducted to illustrate the proposed method. The results show that the proposed method has the ability to discriminate DEA-efficient from DEA-inefficient DMUs and rank DMUs from a more neutral perspective. In addition, the gap minimization criterion to reduce extreme peer-evaluation results and the weights of the cross-efficiency positively influenced by the DEA-efficiency scores of the currently evaluated DMU are also beneficial to conflict mitigation. The results also indicate that the proposed method is compatible with typical cross-efficiency models and makes valid contributions to cross-efficiency evaluation. Future work may focus on how to determine the importance weight $\rho$ of the gap minimization goal in different application contexts.

\section{Conflict of Interests}

All of the authors declare that there is no conflict of interests regarding the publication of this paper.

\section{Acknowledgments}

The authors are grateful to the editor and the anonymous referees for their valuable and constructive comments. This 
work is partially supported by the Fundamental Research Funds for the Central Universities, HUST (CXY13Q035, CXY12Q044), and the Graduates' Innovation Fund of Huazhong University of Science \& Technology (HF-11-202013). The corresponding author thanks the Doctorate Fellowship Foundation of Huazhong University of Science \& Technology and the financial support from China Scholarship Council (201406160046).

\section{References}

[1] A. Charnes, W. W. Cooper, and E. Rhodes, "Measuring the efficiency of decision making units," European Journal of Operational Research, vol. 2, no. 6, pp. 429-444, 1978.

[2] R. D. Banker, A. Charnes, and W. W. Cooper, "Some models for estimating technical a nd scale inefficiencies in data envelopment analysis," Management Science, vol. 30, no. 9, pp. 10781092, 1984.

[3] A. Ebrahimnejad, M. Tavana, F. Hosseinzadeh-Lotfi, R. Shahverdi, and M. Yousefpour, "A three-stage data envelopment analysis model with application to banking industry," Journal of Basic and Applied Scientific Research, vol. 49, pp. 308-319, 2014.

[4] M. Khodabakhshi and K. Aryavash, "The fair allocation of common fixed cost or revenue using DEA concept," Annals of Operations Research, vol. 214, pp. 187-194, 2014.

[5] S. G. Jalali Naini and H. R. Nouralizadeh, "A two-stage DEA to analyze the effect of entrance deregulation on Iranian insurers: a robust approach," Mathematical Problems in Engineering, vol. 2012, Article ID 423524, 24 pages, 2012.

[6] A. Payan, A. A. Noora, F. Hosseinzadeh-Lotfi, and A. Khodabakhshi, "Relative efficiency in two-stage dea and its application to bank branches," Journal of Basic and Applied Scientific Research, vol. 3, pp. 396-404, 2013.

[7] P. Andersen and N. Petersen, "A procedure for ranking efficient units in data envelopment analysis," Management Science, vol. 39, pp. 1261-1264, 1993.

[8] H. Bal and H. H. Örkcü, "A goal programming approach to weight dispersion in data envelopment analysis," Gazi University Journal of Science, vol. 20, pp. 117-125, 2007.

[9] H. Bal, H. H. Örkcü, and S. Çelebioğlu, "Improving the discrimination power and weights dispersion in the data envelopment analysis," Computers and Operations Research, vol. 37, no. 1, pp. 99-107, 2010.

[10] Y. Chen, "Measuring super-efficiency in DEA in the presence of infeasibility," European Journal of Operational Research, vol. 161, no. 2, pp. 545-551, 2005.

[11] F. Hosseinzadeh-Lotfi, G. R. Jahanshahloo, and M. Mohammadpour, "An extension of cross redundancy of interval scale outputs and inputs in DEA," Journal of Applied Mathematics, vol. 2013, Article ID 658635, 7 pages, 2013.

[12] M. Khodabakhshi, M. M. Goudarzi, M. Y. Maryaki, and M. Hajiani, "A one-model approach for computation of congestion with productions trade-offs and weight restrictions," International Journal of Applied Mathematics, vol. 3, pp. 69-80, 2013.

[13] S. Li, G. R. Jahanshahloo, and M. Khodabakhshi, "A superefficiency model for ranking efficient units in data envelopment analysis," Applied Mathematics and Computation, vol. 184, no. 2, pp. 638-648, 2007.

[14] H. H. Örkcü and H. Bal, "Goal programming approaches for data envelopment analysis cross efficiency evaluation," Applied Mathematics and Computation, vol. 218, no. 2, pp. 346-356, 2011.
[15] S. Saati, M. Zarafat, A. Memariani, and G. R. Jahanshahloo, "A model for ranking decision making units in data envelopment analysis," Ricerca Operativa, vol. 31, pp. 47-59, 2001.

[16] Y. Wang and K. Chin, "A neutral DEA model for cross-efficiency evaluation and its extension," Expert Systems with Applications, vol. 37, no. 5, pp. 3666-3675, 2010.

[17] Y. Wang, K. Chin, and P. Jiang, "Weight determination in the cross-efficiency evaluation," Computers and Industrial Engineering, vol. 61, no. 3, pp. 497-502, 2011.

[18] T. R. Sexton, R. H. Silkman, and A. J. Hogan, "Data envelopment analysis: critique and extensions," in Measuring Efficiency: An Assessment of Data Envelopment Analysis, H. R. Silkman, Ed., pp. 73-105, Jossey-Bass, San Francisco, Calif, USA, 1986.

[19] F. Hosseinzadeh Lotfi, G. R. Jahanshahloo, M. Khodabakhshi, M. Rostamy-Malkhlifeh, Z. Moghaddas, and M. Vaez-Ghasemi, "A review of ranking models in data envelopment analysis," Journal of Applied Mathematics, vol. 2013, Article ID 492421, 20 pages, 2013.

[20] J. Doyle and R. Green, "Efficiency and cross-efficiency in DEA: derivations, meanings and uses," Journal of the Operational Research Society, vol. 45, no. 5, pp. 567-578, 1994.

[21] G. R. Jahanshahloo, F. H. Lotfi, Y. Jafari, and R. Maddahi, "Selecting symmetric weights as a secondary goal in DEA cross-efficiency evaluation," Applied Mathematical Modelling: Simulation and Computation for Engineering and Environmental Systems, vol. 35, no. 1, pp. 544-549, 2011.

[22] F. Hosseinzadeh-Lotfi, G. R. Jahanshahloo, and P. Zamani, "A new ranking method based on cross-efficiency in data envelopment analysis," African Journal of Business Management, vol. 5, pp. 7923-7930, 2011.

[23] X. Li and G. R. Reeves, "Multiple criteria approach to data envelopment analysis," European Journal of Operational Research, vol. 115, no. 3, pp. 507-517, 1999.

[24] L. Liang, J. Wu, W. D. Cook, and J. Zhu, "Alternative secondary goals in DEA cross-efficiency evaluation," International Journal of Production Economics, vol. 113, no. 2, pp. 1025-1030, 2008.

[25] C. Tofallis, "Input efficiency profiling: an application to airlines," Computers and Operations Research, vol. 24, no. 3, pp. 253-258, 1997. 


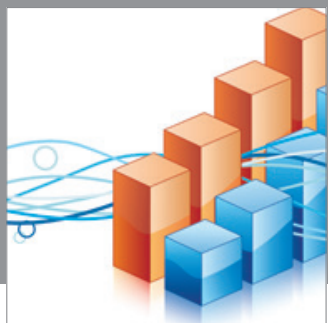

Advances in

Operations Research

mansans

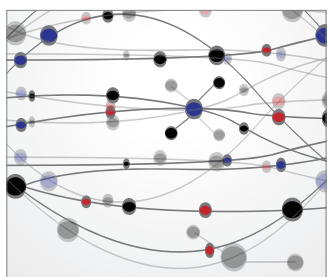

The Scientific World Journal
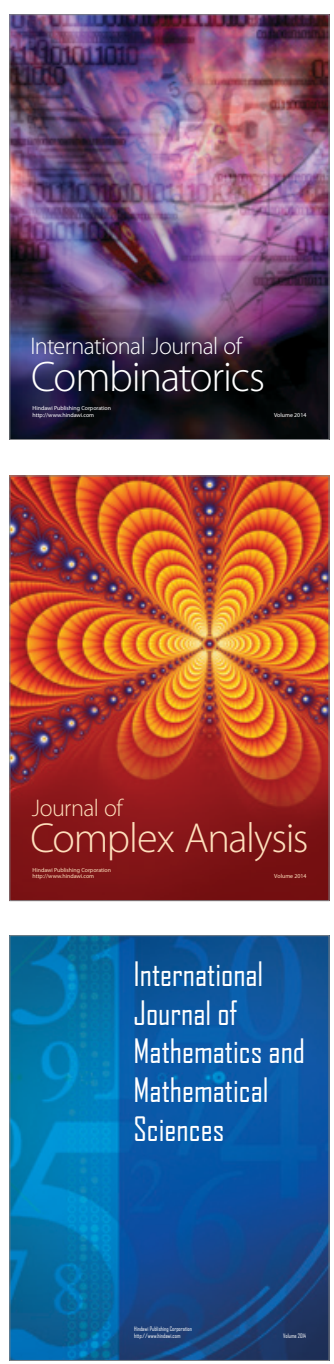
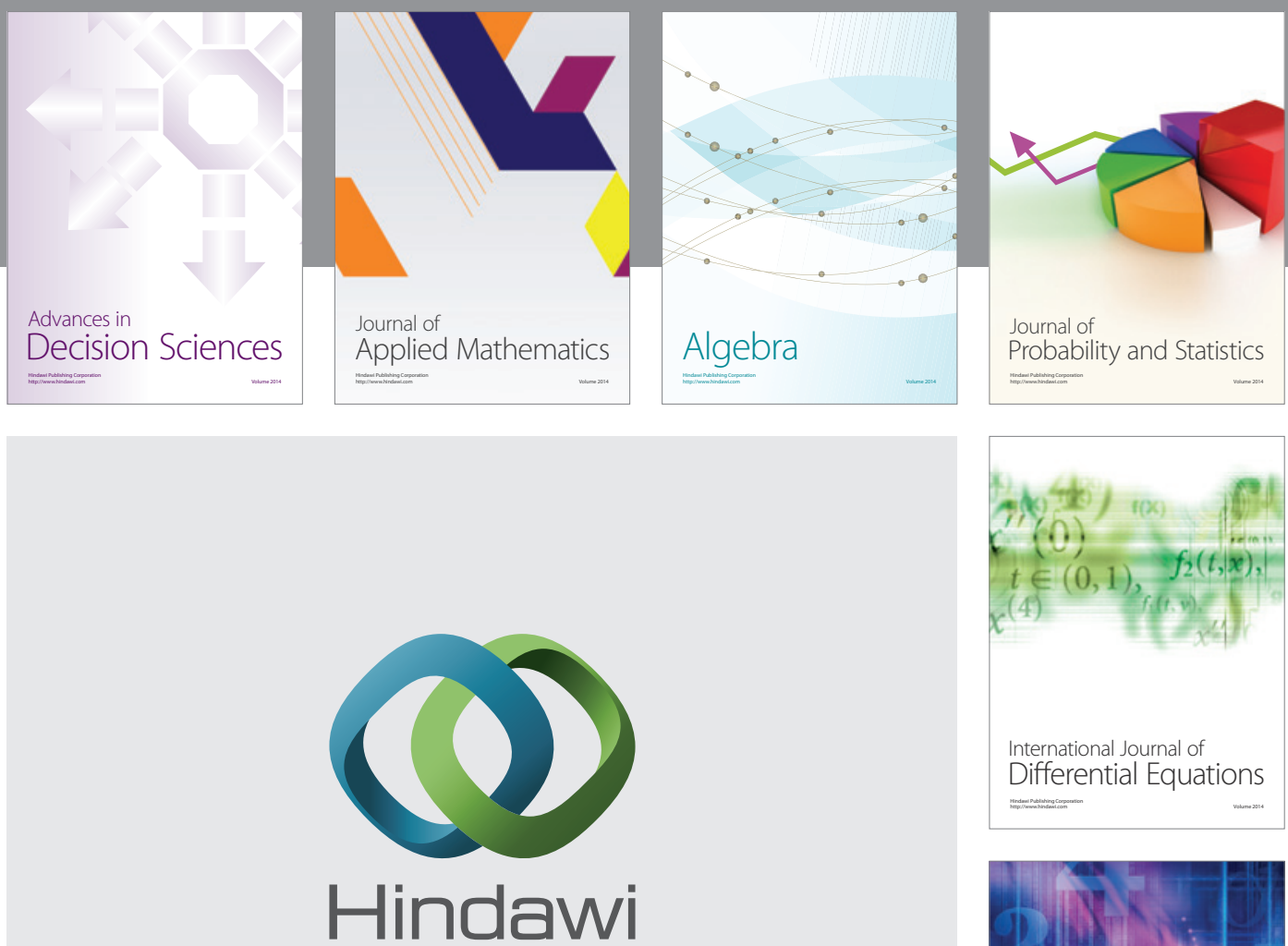

Submit your manuscripts at http://www.hindawi.com
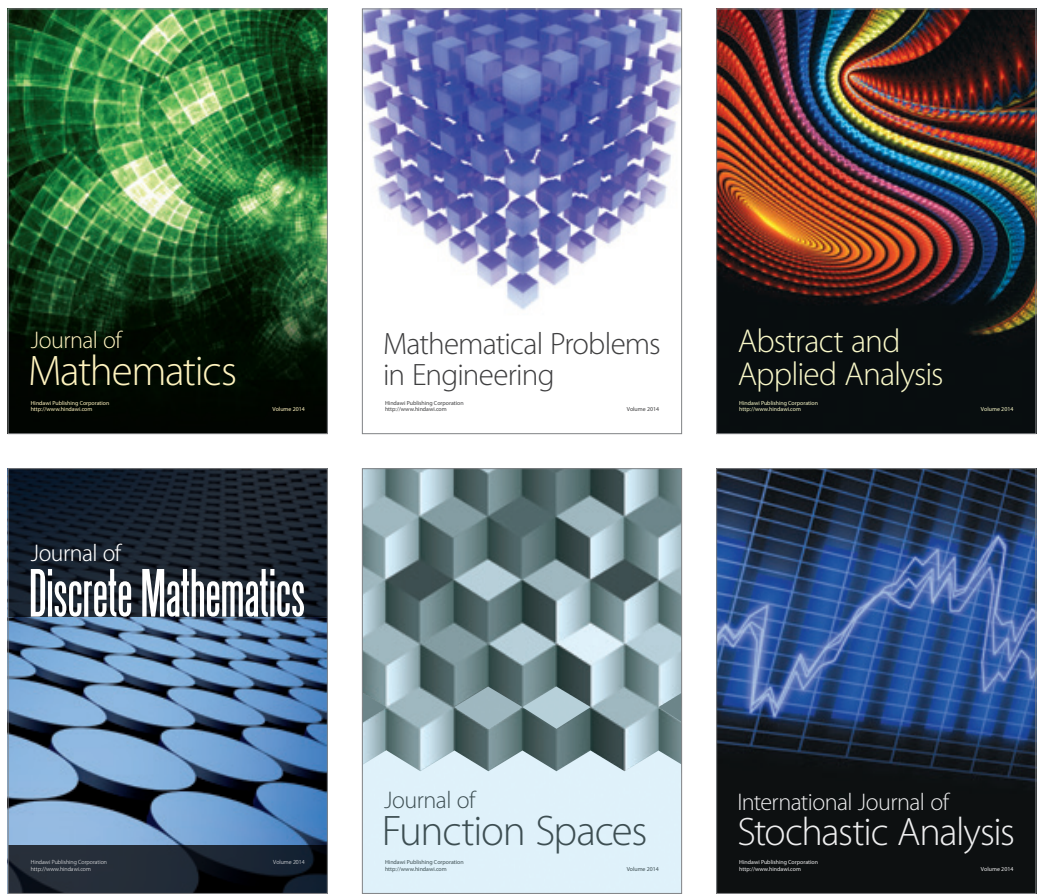

Journal of

Function Spaces

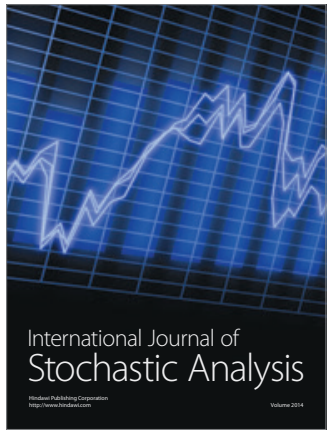

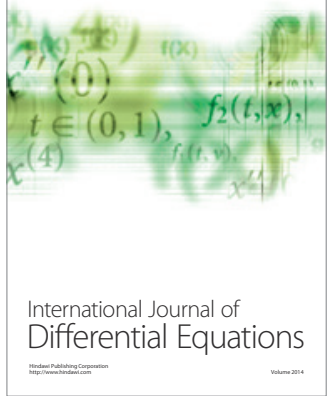
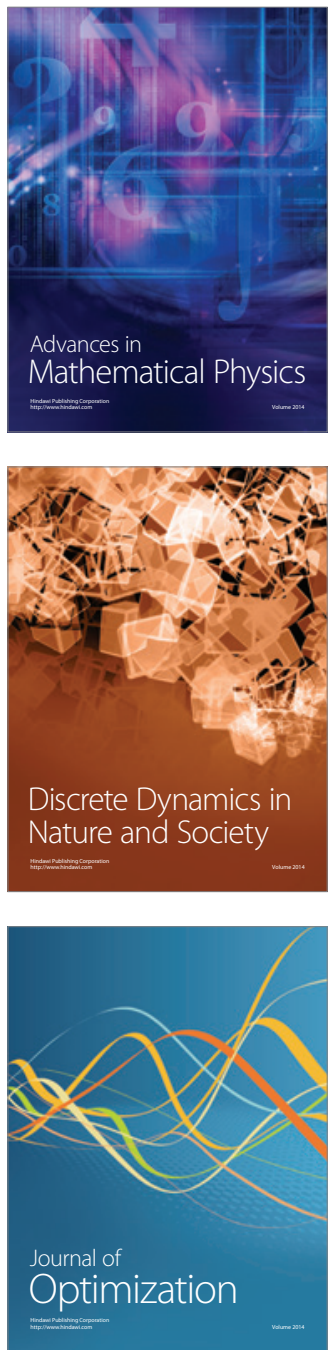